\title{
Pielonefritis xantogranulomatosa en la infancia. A propósito de un caso
}

\author{
R. González Resina, R. Barrero Candau, E. Argüelles Salido, P. Campoy Martínez, \\ A. Rodríguez Pérez, M. Pérez Pérez
}

Servicio de Urología. Hospital Universitario Virgen del Rocío. Sevilla.

Actas Urol Esp 2005; 29 (6): 596-598

\section{RESUMEN}

PIELONEFRITIS XANTOGRANULOMATOSA EN LA INFANCIA. A PROPÓSITO DE UN CASO

La pielonefritis xantogranulomatosa es una rara inflamación crónica del parénquima renal. Predomina en mujeres de edad media y es extremadamente rara en niños.

Presentamos el caso de una niña de 4 años de edad, con infecciones del tracto urinario de repetición y mala respuesta a tratamiento médico. La paciente tenía retraso pondo-estatural y una masa en hemiabdomen izquierdo a la exploración y en las pruebas de imagen. Se realizó heminefrectomía superior izquierda, con posterior confirmación histopatológica de pielonefritis xantogranulomatosa focal.

El tratamiento más frecuente de la forma focal es la nefrectomía parcial, aunque a veces responde a tratamiento antibiótico.

Como conclusión la pielonefritis xantogranulomatosa debe ser considerada dentro de un diagnóstico diferencial de un niño con un riñón aumentado de tamaño e infecciones recurrentes.

Palabras clave: Enfermedad renal. Infancia. Infección del tracto urinario recurrente. Pielonefritis xantogranulomatosa.

\section{ABSTRACT}

XANTHOGRANULOMATOUS PYELONEPHRITIS IN CHILDHOOD. A CASE REPORT.

Xanthogranulomatous pyelonephritis is an uncommon chronic inflammatory renal disorder. Most cases have been described in middle aged women and it is extremely rare in children.

We report a case of a 4 years old girl who suffered from recurrent urinary tract infection and antibiotic therapy resistance. She had low growth-rate and palpable left renal mass on examination and was confirmed by radiological findings. We performed left kidney partial resection and then histological examination showed focal xanthogranulomatous pyelonephritis.

The focal form of the disease may respond to antibiotic treatment although usually an enucleation or partial resection must be performed.

In conclusion xanthogranulomatous pyelonephritis should be considered in the differential diagnosis of a renal mass and recurrent urinary tract infection in childhood.

Keywords: Childhood. Kidney disease. Recurrent urinary tract infection. Xanthogranulomaous pyelonephritis.

La pielonefritis xantogranulomatosa es una rara inflamación crónica del parénquima renal. Descrita por primera vez en 1916 por Schlangenhaufer; Oberling acuñó el término en $1935^{1}$.

Predomina en las mujeres de edad media, suele asociarse a litiasis y es extremadamente rara en niños. Avent publicó el primer caso de pielonefritis xantogranulomatosa infantil en 1963. Existen dos tipos, difusa y focal siendo esta última la que se encuentra con mayor frecuencia en los pocos casos descritos en niños ${ }^{2,3}$.

Presentamos un caso de pielonefritis xantogranulomatosa focal en una niña.

\section{CASO CLINICO}

Niña de 4 años que consulta por infecciones del tracto urinario de repetición de un año de evolución, causada por gérmenes habituales y 
con mala respuesta a las diferentes pautas antibióticas prescritas.

A la exploración física se observa un retraso pondo-estatural discreto y empastamiento en hemiabdomen izquierdo.

En la analítica destaca una leve anemia normocrómica normocítica, microhematuria y leucocituria. La ecografía revela un aumento global del riñón izquierdo y sospecha de masa en su polo superior. La urografía intravenosa confirma los hallazgos de la ecografía, con retraso en la captación y eliminación del contraste en la unidad renal alterada. La cistouretrografia miccional seriada fue normal.

La gammagrafía renal muestra una lesión ocupante de espacio en tercio superior del riñón izquierdo (Fig. 1), y la arteriografía renal selectiva fue compatible con displasia renal (Fig. 2).

Sin diagnóstico preoperatorio de certeza se realiza heminefrectomía superior renal izquierda con biopsia intraoperatoria de pielonefritis xantogranulomatosa, diagnóstico que fue confirmado por el estudio histopatológico definitivo.

La paciente tuvo un postoperatorio sin complicaciones y vive libre de infecciones y con un desarrollo normal.

\section{DISCUSIÓN}

Han sido propuestas numerosas teorías para aclarar la etiología de la pielonefritis xantogranulomatosa, obstrucción del tracto urinario, infecciones de repetición o un metabolismo alterado; sin embargo, la causa exacta aún es desconocida ${ }^{2}$.

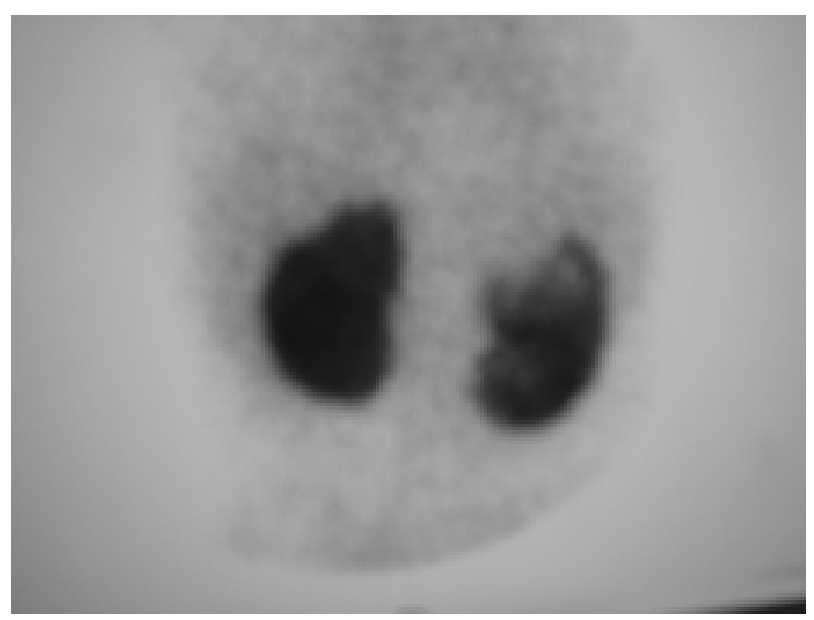

FIGURA 1. Gammagrafia renal: tercio superior de riñón izquierdo con parénquima adelgazado pero funcionante, con sospecha de lesión ocupante de espacio.

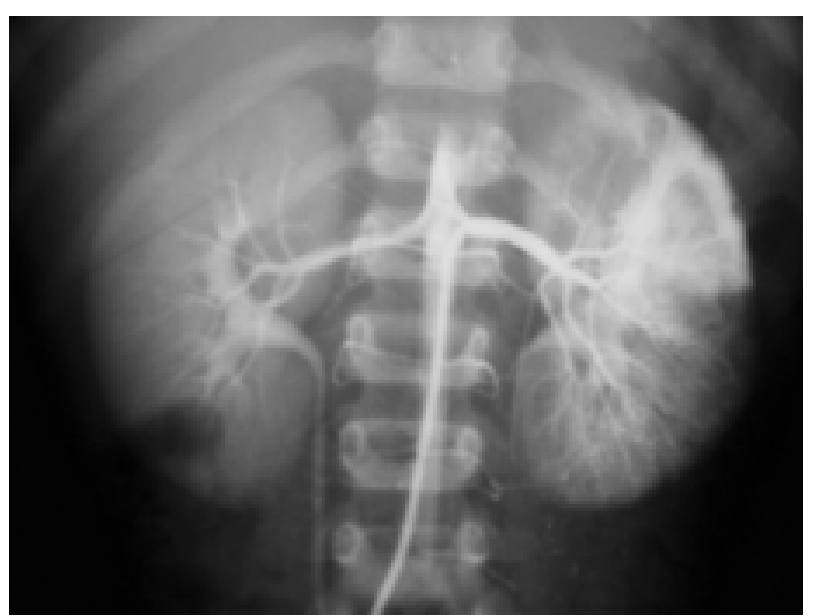

FIGURA 2. Arteriografia renal selectiva: sospecha de displasia del polo superior renal izquierdo.

Esta entidad debe ser sospechada ante la presencia de un niño con una historia de infecciones de repetición resistentes a los antibióticos, cálculos renales, riñón aumentado de tamaño con función empobrecida, anemia, leucocitosis y microhematuria ${ }^{2,4}$.

La ecografía puede mostrar los signos patognomónicos de pielonefritis xantogranulomatosa que son cálculo renal con ecogenicidad central, riñón aumentado de tamaño con áreas multifocales de densidad variada e infiltración de los tejidos perirrenales. Nuestro caso no presentaba estas características si bien es cierto que el polo superior era difícilmente explorable por interposición costal.

La tomografía computerizada (TC) puede ayudar al diagnóstico diferencial preoperatorio con el tumor de wilms. Cuando nuestra niña fue dignosticada, la TC no estaba desarrollada en nuestro centro, por ello fue sometida a una arteriografía, prueba que hoy día habitualmente no es precisa ${ }^{5}$.

El examen histológico suele mostrar colecciones de macrófagos con cristales de colesterol, fibrosis y un infiltrado inflamatorio; la mayoría de las series muestran predisposición por el lado izquierdo ${ }^{6}$; algunos autores consideran que existe un aumento en la incidencia de pielonefritis difusa en detrimento de la focal en niños en proporción de 9: $1^{2}$.

La forma focal de pielonefritis xantogranulomatosa puede ser tratada en casos seleccionados con antibioterapia, no obstante es vital la confirmación anatomopatológica de la enfermedad tan pronto como sea posible. La enucleación o nefrec- 
tomía parcial es el tratamiento más frecuente, aunque no son pocos los casos que son diagnosticados tras una nefrectomía ${ }^{2,4}$.

El pronóstico suele ser excelente tal y como ocurrió en nuestro caso.

Como conclusión diremos que la pielonefritis xantogranulomatosa debe ser considerada dentro de un diagnóstico diferencial de un niño con un riñón aumentado de tamaño e infecciones recurrentes.

\section{REFERENCIAS}

1. Hammadeh MY, Nicholls CJ, Calder RG, Buick P, Gornall P, Corkery JJ. Xanthogranulomatous pyelonephritis in childhood: pre-operative diagnosis is possible. $\mathrm{Br} \mathrm{J}$ Urol 1994;73(1):83-86.

2. TakamizawA S, Yamataka A, Kaneko K, Yanai T, Yamashiro Y, Miyano T. Xanthogranulomatous pyelonephritis in Childhood: A rare but important clinical entity. J Pediatr Surg 2000;35(11):1554-1555.
3. Cakmakci H, Tasdelen N, Obuz F, Yilmaz E, Kovanlikaya A. Pediatric focal xanthogranulomatous pyelonephritis: dynamic contrast-enhanced MRI findings. Clin Imaging 2002;26(3): 183-186

4. Samuel M, Duffy P, Capps S, Mouriquand P, Williams D, Ransley P. Xanthogranulomatous pyelonephritis in childhood. J Pediatr Surg 2001;36(4):598-601.

5. Shanser JD,Herzog KA, Palubinskas AJ. Xanthogranulomatous pyelonephritis in childhood. Pediatr Radiol 1975;3(1): 12-15.

6. Guinn FM, Dick AC, Corbally MT, Mcdermott MB, Guinev EJ. Xanthogranulomatous pyelonephritis in childhood. Arch Dis Child 1999;81(6):483-486.

Dra. R. González Resina.

C/ Cardenal Ilundain 24, Esc. 4, 2ㅇ C

41013 Sevilla

(Trabajo recibido el 8 octubre 2004) 